\title{
ASYMPTOTIC BEHAVIOR OF THE SOLUTIONS OF A DISCRETE REACTION-DIFFUSION EQUATION
}

\author{
RIGOBERTO MEDINA and SUI SUN CHENG
}

Received 3 April 2001 and in revised form 12 July 2001

\begin{abstract}
By means of Bihari type inequalities, we derive sufficient conditions for solutions of a discrete reaction-diffusion equation to be bounded or to converge to zero. Asymptotic representation of solutions are also derived. Our results yield estimates and explicit attractive regions for the solutions.
\end{abstract}

2000 Mathematics Subject Classification: 39A10.

1. Introduction. Discrete reaction-diffusion type partial difference equations have recently been introduced by a number of authors as models for the study of spatiotemporal chaos (cf. [4]). Stability criteria have also been derived for such equations, which involves two-level (see [2]) as well as three-level processes (cf. [3]). Besides, the question of stability and asymptotic representation of solutions of such difference schemes are also of fundamental importance. In this paper, we study nonlinear twolevel partial difference equations and, by means of comparison theorems, we derive sufficient conditions for the solutions to be bounded or to converge to zero. Further, asymptotic formulae for the solutions of the above equation are obtained. We want to point out that our results compare favorably with results in [1, 2], basically because their main results are valid only for linear or sublinear perturbations. Besides, our results are new and do not overlap with those in [1, 2].

2. Preliminary facts. Let $\mathbb{R}$ be the set of reals and $\mathbb{N}$ the set of nonnegative integers. Consider a discrete reaction-diffusion equation of the form

$$
u_{i}^{(j+1)}=a u_{i-1}^{(j)}+b u_{i}^{(j)}+c u_{i+1}^{(j)}+g_{i}^{(j)}+\tilde{F}\left(i, j, u_{i}^{(j)}\right),
$$

where $i=1,2, \ldots, n ; j \in \mathbb{N} ; a, b, c \in \mathbb{R} ; g=\left\{g_{i}^{(j)}\right\}$ is a real function defined for $i=$ $1,2, \ldots, n$ and $j \in \mathbb{N}$, and $\tilde{F}$ is a real function. We will also assume that side conditions

$$
\begin{aligned}
u_{0}^{(j)}=h_{j} \in \mathbb{R}, & j \in \mathbb{N}, \\
u_{n+1}^{(j)}=q_{j} \in \mathbb{R}, & j \in \mathbb{N}, \\
u_{i}^{(0)}=\tau_{i} \in \mathbb{R}, & i=1,2, \ldots, n,
\end{aligned}
$$

are imposed. Let

$$
\Omega=\{(i, j) \mid i=0,1, \ldots, n+1 ; j \in \mathbb{N}\}
$$


A solution of (2.1) and (2.2) is a discrete function $u=\left\{u_{i}^{(j)}\right\}_{(i, j) \in \Omega}$ which satisfies the functional relation (2.1) and also the side conditions (2.2). If we put $u^{(j)}=\operatorname{col}\left(u_{1}^{(j)}\right.$, $\left.u_{2}^{(j)}, \ldots, u_{n}^{(j)}\right)$ and $\tau=\operatorname{col}\left(\tau_{1}, \ldots, \tau_{n}\right)$, then the sequence $\left\{u^{(j)}\right\}_{j=0}^{\infty}$ will satisfy the twoterm vector equation

$$
u^{(j+1)}=A u^{(j)}+f_{j}+F\left(j, u^{(j)}\right), \quad j \in \mathbb{N},
$$

subject to the initial condition

$$
u^{(0)}=\tau,
$$

where

$$
\begin{gathered}
A=\left[\begin{array}{cccccc}
b & c & 0 & \ldots & \ldots & 0 \\
a & b & c & 0 & \ldots & 0 \\
0 & a & b & c & \ldots & 0 \\
\ldots & \ldots & \ldots & \ldots & \ldots & \ldots \\
\ldots & \ldots & \ldots & \ldots & \ldots & \ldots \\
0 & \ldots & \ldots & 0 & a & b
\end{array}\right], \\
f_{j}=\left(g_{1}^{(j)}, \ldots, g_{n}^{(j)}\right)+\operatorname{col}\left(a h_{j}, 0, \ldots, 0, c q_{j}\right), \\
F(j, x)=\operatorname{col}\left(\tilde{F}\left(1, j, x_{1}\right), \ldots, \tilde{F}\left(n, j, x_{n}\right)\right) .
\end{gathered}
$$

Conversely, if $\left\{u^{(j)}\right\}_{j=0}^{\infty}$ is a solution of (2.4) and (2.5), then by augmenting each $u^{(j)}=$ $\operatorname{col}\left(u_{1}^{(j)}, \ldots, u_{n}^{(j)}\right)$ with the terms $u_{0}^{(j)}=h_{j}$ and $u_{n+1}^{(j)}=q_{j}$ to form $\left\{u_{0}^{(j)}, u_{1}^{(j)}, \ldots, u_{n}^{(j)}\right.$, $u_{n+1}^{(j)}$, we see that the resulting family forms a solution of (2.1) and (2.2).

THEOREM 2.1 (see [5]). Let $B=\left(b_{i j}\right)_{n \times n}$ be a real matrix and $\rho(B)$ its spectral radius. Then, there exists a constant $\Gamma \geq 1$, such that $\left\|B^{i}\right\| \leq \Gamma(\rho(B))^{i}$ for $i \in \mathbb{N}$.

We will use the following theorem which gives an explicit estimate for a function $u=u(m)$ which satisfies the functional inequality

$$
u(m) \leq c+\sum_{i=1}^{p} \sum_{j=0}^{m-1} \lambda_{i}(j) w_{i}(u(j)), \quad m \in \mathbb{N},
$$

where (G1) $c \geq 0, p$ is a positive integer, (G2) $\lambda_{1}, \lambda_{2}, \ldots, \lambda_{p}$ are nonnegative sequences in $\ell_{1}(\mathbb{N})$, where $\ell_{1}(\mathbb{N})$ is the set of all absolutely summable real sequences defined on $\mathbb{N}$, and (G3) the functions $w_{i}, 1 \leq i \leq p$, are continuous on $[0, \infty)$ and positive on $(0, \infty)$, such that $w_{i+1} / w_{i}, 1 \leq i \leq p-1$, are nondecreasing on $(0, \infty)$.

To this end, we need to define

$$
\begin{aligned}
W_{i}(u) & =\int_{u_{i}}^{u} \frac{1}{w_{i}(s)} d s, \quad u>0, u_{i}>0,1 \leq i \leq p, \\
\Psi_{i}(u) & =W_{i}^{-1}\left(W_{i}(u)+\alpha_{i}\right),
\end{aligned}
$$

with $\alpha_{1}, \ldots, \alpha_{p} \in \mathbb{R}$, and $\varphi_{0}(u)=u, \varphi_{i}=\Psi_{i} \circ \Psi_{i-1} \circ \cdots \circ \Psi_{1}$ for $1 \leq i \leq p$. 
TheOREM 2.2 (see [6, 7]). Under the conditions (G1), (G2), and (G3), if $u=\{u(m)\}_{m \in \mathbb{N}}$ is a nonnegative sequence which satisfies (2.7), $\alpha_{i}=\sum_{j=0}^{\infty} \lambda_{i}(j)$, and $c<\varphi_{p}^{-1}(\infty)$, then

$$
u(m) \leq W_{p}^{-1}\left(W_{p}\left(\varphi_{p-1}(c)\right)+\sum_{k=0}^{m-1} \lambda_{p}(k)\right), \quad m \in \mathbb{N},
$$

and $u(m) \leq \varphi_{p}(c)$ for $m \in \mathbb{N}$.

We remark that if

$$
\int_{1}^{\infty} \frac{d s}{w_{i}(s)}=\infty, \quad 1 \leq i \leq p,
$$

holds, then the same conclusion $u(m) \leq \varphi_{p}(c)$ is valid for all $c>0$. If the dual condition

$$
\int_{0+}^{1} \frac{d s}{w_{i}(s)}=\infty, \quad 1 \leq i \leq p,
$$

holds, then the same conclusion $u(m) \leq \varphi_{p}(c)$ is valid if $c$ is small enough.

EXAMPLE 2.3. Let $w_{i}(u)=u^{n_{i}}$ for $i=1,2$. Then

$$
\Psi_{i}(u)= \begin{cases}{\left[u^{1-n_{i}}+\alpha_{i}\left(1-n_{i}\right)\right]^{-1 /\left(n_{i}-1\right)},} & n_{i} \in(0,1) \cup(1, \infty), \\ u \exp \alpha_{i}, & n_{i}=1,\end{cases}
$$

for $i=1,2$. Thus, for $n_{i} \neq 1, \varphi=\Psi_{2} \circ \Psi_{1}$ takes the form

$$
\varphi(u)=\left\{\left[u^{1-n_{1}}+\alpha_{1}\left(1-n_{1}\right)\right]^{\left(n_{2}-1\right) /\left(n_{1}-1\right)}+\alpha_{2}\left(1-n_{2}\right)\right\}^{-1 /\left(n_{2}-1\right)} .
$$

Moreover, since $\Psi_{i}^{-1}(u)=W_{i}^{-1}\left[W_{i}(u)-\alpha_{i}\right], \varphi^{-1}(u)$ is obtained from (2.13) by replacing $\alpha_{i}$ with $-\alpha_{i}$. Thus, for $n_{i}>1, \varphi(u)$ is defined for all $u<\varphi^{-1}(\infty)$, where

$$
\varphi^{-1}(\infty)=\left\{\left[\alpha_{1}\left(n_{1}-1\right)\right]^{\left(n_{2}-1\right) /\left(n_{1}-1\right)}+\alpha_{2}\left(n_{2}-1\right)\right\}^{-1 /\left(n_{2}-1\right)} .
$$

For the case $n_{1}=1<n_{2}$, we get

$$
\begin{aligned}
\varphi(u) & =\left[\left(u e^{\alpha_{1}}\right)^{1-n_{2}}+\alpha_{2}\left(1-n_{2}\right)\right]^{-1 /\left(n_{2}-1\right)} \\
& =u e^{\alpha_{1}}\left[1+\alpha_{2}\left(1-n_{2}\right)\left(u e^{\alpha_{1}}\right)^{n_{2}-1}\right]^{-1 /\left(n_{2}-1\right)}
\end{aligned}
$$

which is defined for

$$
u<\varphi^{-1}(\infty)=\left[\alpha_{2}\left(n_{2}-1\right)\right]^{-1 /\left(n_{2}-1\right)},
$$

and if $n_{1}<1=n_{2}$, then

$$
\varphi(u)=e^{\alpha_{2}}\left[u^{1-n_{1}}+\alpha_{1}\left(1-n_{1}\right)\right]^{-1 /\left(n_{1}-1\right)}
$$

is defined for $u<\varphi^{-1}(\infty)=(\infty)$. 
3. Boundedness. Now, we can establish our boundedness criteria.

THEOREM 3.1. Assume that

$$
\|F(j, x)\| \leq \sum_{l=1}^{p} \lambda_{l}(j) w_{l}(\|x\|), \quad j \in \mathbb{N},
$$

for all $x \in \mathbb{R}^{n}$, where $w_{1}, \ldots, w_{p}$ satisfy condition (G3), and $\lambda_{1}, \ldots, \lambda_{p}$ are nonnegative sequences in $\ell_{1}(\mathbb{N})$. In addition, suppose $\sum_{k=0}^{\infty}\left\|f_{k}\right\|<\infty, \rho(A)=\rho \leq 1$, and $\left\|A^{i}\right\| \leq \Gamma \rho^{i}$ for $i \in \mathbb{N}$. If there exists a constant $d>0$ such that

$$
\sum_{k=0}^{\infty} \lambda_{l}(k)<\frac{1}{\Gamma} \int_{\varphi_{l-1}(d)}^{\infty} \frac{1}{w_{l}(s)} d s, \quad 1 \leq l \leq p,
$$

and $\Gamma\left(\|\boldsymbol{T}\|+\sum_{k=0}^{\infty}\left\|f_{k}\right\|\right)<d$, then all solutions of (2.4) are bounded.

Proof. By inductive arguments, it is easily seen that the unique solution $\left\{u^{(j)}\right\}_{j=0}^{\infty}$ of (2.4), subject to $u^{(0)}=\tau$, satisfies

$$
u^{(j)}=A^{j} \boldsymbol{\tau}+\sum_{k=0}^{j-1} A^{j-k-1} f_{k}+\sum_{k=0}^{j-1} A^{j-k-1} F\left(k, u^{(k)}\right), \quad j \in \mathbb{N} .
$$

Thus, in view of Theorem 2.1 and our assumptions,

$$
\left\|u^{(j)}\right\| \leq \Gamma \rho^{j}\|\tau\|+\Gamma \sum_{k=0}^{j-1} \rho^{j-k-1}\left\|f_{k}\right\|+\Gamma \sum_{l=1}^{p} \sum_{k=0}^{j-1} \rho^{j-k-1} \lambda_{l}(k) w_{l}\left(\left\|u^{(k)}\right\|\right),
$$

where $\Gamma$ is some positive number greater than or equal to 1 . Put $v(j)=\left\|u^{(j)}\right\|$ for $j \in \mathbb{N}$. Then

$$
\begin{aligned}
v(j) & \leq \Gamma\|\tau\|+\Gamma \sum_{k=0}^{\infty}\left\|f_{k}\right\|+\Gamma \sum_{l=1}^{p} \sum_{k=0}^{j-1} \lambda_{l}(k) w_{l}(v(k)) \\
& \leq d+\Gamma \sum_{l=1}^{p} \sum_{k=0}^{j-1} \lambda_{l}(k) w_{l}(v(k)) .
\end{aligned}
$$

So, by Theorem 2.2, we obtain

$$
v(j) \leq W_{p}^{-1}\left(W_{p}\left(\varphi_{p-1}(d)\right)+\Gamma \sum_{k=0}^{j-1} \lambda_{p}(k)\right), \quad j \in \mathbb{N} .
$$

Inequalities (3.2) show that this estimation is valid for all $j \in \mathbb{N}$, and that the function in the right-hand side is bounded, in fact,

$$
W_{p}^{-1}\left(W_{p}\left(\varphi_{p-1}(d)\right)+\Gamma \sum_{k=0}^{\infty} \lambda_{p}(k)\right) \leq \varphi_{p}(d) .
$$

Hence,

$$
v(j) \leq \varphi_{p}(d) ; \quad j \in \mathbb{N}
$$

concluding the proof. 
REMARKS. (a) If $\int_{1}^{\infty}\left(1 / w_{l}(s)\right) d s=\infty, 1 \leq l \leq p$, then conditions (3.2) of Theorem 3.1 are satisfied for all $d>0$.

(b) If $\int_{0+}^{1}\left(1 / w_{l}(s)\right) d s=\infty, 1 \leq l \leq p$, then there always exists a small $d$ satisfying conditions (3.2) of Theorem 3.1.

(c) If $\int_{0+}^{1}\left(1 / w_{l}(s)\right) d s<\infty, 1 \leq l \leq p$, then the inequality $\sum_{k=0}^{\infty} \lambda_{l}(k) \geq \int_{0}^{\infty}\left(1 / w_{l}(s)\right) d s$ for some $l, 1 \leq l \leq p$, implies that there is no $d>0$ satisfying (3.2). In every case, the biggest $d$ satisfying conditions (3.2) is $d=\varphi_{p}^{-1}(\infty)$.

Thus, we can establish the following corollary of Theorem 3.1.

COROLlary 3.2. (A) If $\int_{1}^{\infty}\left(1 / w_{l}(s)\right) d s=\infty, 1 \leq l \leq p$, holds, then the result of Theorem 3.1 is valid for all solutions.

(B) If $\int_{0+}^{1}\left(1 / w_{l}(s)\right) d s=\infty, 1 \leq l \leq p$, holds, then the statement of Theorem 3.1 is valid for all solutions of (2.4) such that $\Gamma\left(\left\|u^{(0)}\right\|+\sum_{k=0}^{\infty}\left\|f_{k}\right\|\right)$ is small enough, namely, $\Gamma\left(\left\|u^{(0)}\right\|+\sum_{k=0}^{\infty}\left\|f_{k}\right\|\right)<\varphi_{p}^{-1}(\infty)$.

COROLlary 3.3. Suppose that

$$
\|F(j, x)\| \leq \sum_{l=1}^{p} \lambda_{l}(j)\|x\|^{\eta_{l}}, \quad j \in \mathbb{N},
$$

where $\eta_{i} \geq 1,1 \leq i \leq p$ and $\lambda_{1}, \ldots, \lambda_{p}$ are positive sequences in $\ell_{1}(\mathbb{N})$. Then, the statement of Theorem 3.1 is valid for all sufficiently small positive $d$.

REMARK 3.4. If $0<\eta_{i}<1,1 \leq i \leq p$, in (3.9), then the statement of Theorem 3.1 remains valid for all $d>0$, and consequently for all solutions of (2.4).

THEOREM 3.5. Suppose that the function $F=F(j, x)$ satisfies (3.9), where

(I) the functions $w_{l}, 1 \leq l \leq p$, satisfy conditions (G3), and for any $l, 1 \leq l \leq p$, there is a function $r_{l}$ defined on $(0, \infty)$ such that $w_{l}(\alpha u) \leq r_{l}(\alpha) w_{l}(u)$ for $\alpha \geq 0, u \geq 0$;

(II) the functions $\lambda_{l}, 1 \leq l \leq p$, satisfy $\sum_{k=0}^{\infty} \rho^{-k} \cdot \lambda_{l}(k) r_{l}\left(\rho^{k}\right)<\infty$;

(III) there is a constant $d>0$ such that

$$
\sum_{k=0}^{\infty} \rho^{-k} \cdot \lambda_{l}(k) r_{l}\left(\rho^{k}\right)<\frac{\rho}{\Gamma} \int_{\varphi_{l-1}(d)}^{\infty} \frac{d s}{w_{l}(s)}, \quad 1 \leq l \leq p ;
$$

(IV) $\sum_{k=0}^{\infty} \rho^{-k}\left\|f_{k}\right\|<\infty$. If $\rho(A)=\rho<1$ and $\left\|A^{i}\right\| \leq \Gamma \rho^{i}$ for $i \in \mathbb{N}$, then any solution $u^{(j)}$ of (2.4), such that $\Gamma\left(\|\tau\|+\sum_{k=0}^{\infty} \rho^{-(k+1)}\left\|f_{k}\right\|\right)<d$, converges to zero, as $j \rightarrow \infty$.

Indeed, it suffices to proceed in a way similar to the proof of Theorem 3.1, thus we omit it.

COROLLARY 3.6. Suppose that

$$
\|F(j, x)\| \leq \sum_{l=1}^{p} \lambda_{l}(j)\|x\|^{\eta_{l}}, \quad j \in \mathbb{N},
$$

where $\eta_{i} \geq 1,1 \leq i \leq p$ and the sequences $\lambda_{1}, \ldots, \lambda_{p}$ satisfy

$$
\sum_{k=0}^{\infty} \rho^{-k\left(1-\eta_{l}\right)} \cdot \lambda_{l}(k)<\infty, \quad 1 \leq l \leq p .
$$


Further, suppose that there exists a constant $d>0$ such that

$$
\sum_{k=0}^{\infty} \rho^{-k\left(1-\eta_{l}\right)} \cdot \lambda_{l}(k)<\frac{\rho}{\Gamma} \frac{\left(\varphi_{l-1}(d)\right)^{1-\eta_{l}}}{1-\eta_{l}}, \quad 1 \leq l \leq p .
$$

Then the statement of Theorem 3.5 is valid for all sufficiently small positive $d$.

REMARK 3.7. We want to point out that $\varphi_{p}(d)$ and $\varphi_{p}^{-1}(\infty)$ can be explicitly calculated (see Section 2, Example 2.3), and consequently, we can show the radius of attraction for bounded and convergent to zero solutions (see [6]).

4. Asymptotic representation. In this section, our objective is to obtain asymptotic formulae for the solution of discrete reaction-diffusion equations of the form (2.1), but under the additional conditions $u_{0}^{(j)}=0=u_{n+1}^{(j)} ; j \in \mathbb{N}$ and $g_{i}^{(j)}=0$ for all $i=1,2, \ldots, n$; $j \in \mathbb{N}$.

By iteration and induction, it can be proved that the unique vector solution $\left\{u^{(j)}\right\}_{j=0}^{\infty}$ of the reduced equation

$$
u^{(j+1)}=A u^{(j)}+F\left(j, u^{(j)}\right) ; \quad j \in \mathbb{N},
$$

subject to $u^{(0)}=\tau$, satisfies

$$
u^{(j)}=A^{j} \tau+\sum_{k=0}^{j-1} A^{j-k-1} F\left(k, u^{(k)}\right), \quad j \in \mathbb{N} .
$$

THEOREM 4.1. Assume that $\|F(j, x)\| \leq \lambda(j) w(\|x\|)$, for all $x \in \mathbb{R}^{n}$ and $j \in \mathbb{N}$, where $w:[0, \infty) \rightarrow[0, \infty)$ is a continuous, positive, and nondecreasing function on $[0, \infty)$. Further, suppose that $\lambda \in \ell_{1}(\mathbb{N})$, and there exists a constant $d>0$ such that

$$
\sum_{k=0}^{\infty} \lambda(k)<\frac{1}{\Gamma} \int_{d\|\tau\|}^{\infty} \frac{d s}{w(s)} .
$$

If $\rho(A)=\rho \leq 1$, and $\left\|A^{i}\right\| \leq \Gamma \rho^{i}$ for all $i \in \mathbb{N}$, then corresponding to each bounded solution of (4.1) there is $z_{0} \in \mathbb{R}^{n}$ such that $u^{(j)}=A^{j} z_{0}+\tilde{o}(1)$, as $j \rightarrow \infty$, where o (1) represents a vector function of $j$ which is bounded at infinity.

PRoof. From Theorem 3.1, we infer that all solutions $u^{(j)}$ of (4.1), such that $\Gamma\left\|u^{(0)}\right\|$ $<d$, are bounded. On the other hand, we have

$$
\begin{aligned}
\left\|\sum_{k=0}^{j-1} A^{j-k-1} F\left(k, u^{(k)}\right)\right\| & \leq \sum_{k=0}^{j-1} \Gamma \rho^{j-k-1} \lambda(k) w\left(\left\|u^{(k)}\right\|\right) \\
& \leq \Gamma w(K) \sum_{k=0}^{j-1} \lambda(k) \leq \Gamma w(K) \sum_{k=0}^{\infty} \lambda(k),
\end{aligned}
$$

where $K>0$ is a constant such that $\left\|u^{(j)}\right\| \leq K$, for $j \in \mathbb{N}$. Then, for every solution $u$ of (4.1), we see that the solution $v$ of equation

$$
v^{(j+1)}=A v^{(j)} ; \quad j \in \mathbb{N},
$$


given by

$$
v^{(j)}=u^{(j)}-\sum_{k=0}^{j-1} A^{j-k-1} F\left(k, u^{(k)}\right),
$$

has the property

$$
u^{(j)}=v^{(j)}+\tilde{o}(1), \quad \text { as } j \longrightarrow \infty .
$$

Moreover, if $v^{(j)}$ is a solution of (4.5), then there is $z_{0} \in \mathbb{R}^{n}$ such that

$$
v^{(j)}=A^{j} z_{0}
$$

Therefore,

$$
u^{(j)}=A^{j} z_{0}+\tilde{o}(1), \quad \text { as } j \rightarrow \infty
$$

REMARK 4.2. In Theorem 4.1, we assume that $\|\boldsymbol{\tau}\|$ is small enough so that $W\left(0^{+}\right)=$ $-\infty$, where

$$
W(u)=\int_{u_{0}}^{u} \frac{d s}{w(s)}, \quad u>0, u_{0}>0
$$

in order that $W^{-1}$ has meaning, that is, the inverse function $W^{-1}(v)$ is defined for $v \in\left(0, \delta_{0}\right)$, for $\delta_{0}$ small enough.

COROLLARY 4.3. (I) If (2.10) holds, then the result of Theorem 4.1 is valid for every solution of (4.1).

(II) If (2.11) holds, then the result of Theorem 4.1 is valid for every solution $u^{(j)}$ of (4.1), such that $\Gamma\left\|u^{(0)}\right\|<\varphi_{p}^{-1}(\infty)$.

A more precise asymptotic formula is given in the following theorem.

THEOREM 4.4. Under the hypotheses of Theorem 4.1, if in addition,

$$
\sum_{k=0}^{j-1} A^{j-k-1} F\left(k, u^{(k)}\right) \longrightarrow 0, \quad \text { as } j \longrightarrow \infty,
$$

then

$$
u^{(j)}=A^{j} \cdot z_{0}+o(1), \quad \text { as } j \longrightarrow \infty .
$$

THEOREM 4.5. Under the hypotheses of Corollary 4.3(II). Suppose that $\Phi\left(j, j_{0}\right)=$ $A^{j-j_{0}} ; j \geq j_{0}$, satisfies

$$
\left\|\Phi^{-1}(j+1,0) F(j, \Phi(j, 0) z)\right\| \leq \lambda(j) w(\|z\|),
$$

for $j \in \mathbb{N}$ and $z \in \mathbb{R}^{n}$; and $\lambda \in \ell_{1}(\mathbb{N})$. Then, for every solution $u^{(j)}$ of (4.1), with $\left\|u^{(0)}\right\|$ small enough, there is $z_{0} \in \mathbb{R}^{n}$, such that

$$
u^{(j)}=A^{j}\left[z_{0}+O\left(\sum_{\ell=j}^{\infty} \lambda(l)\right)\right], \quad \text { as } j \longrightarrow \infty .
$$


Proof. Making $u^{(j)}=\Phi(j, 0) z(j)$ in (4.1), we get

$$
z(j)=\tau+\sum_{k=0}^{j-1} A^{-k-1} F\left(k, A^{k} z(k)\right) .
$$

The rest of the proof follows by arguments similar to those in the proof of Theorem 4.1, so we omit it.

ACKNOWLEDGEMENT. This research was supported by Fondecyt under Grant No. 1000023.

\section{REFERENCES}

[1] S. S. Cheng and M. I. Gil', Stability of a time discrete perturbed dynamical systems with delay, Discrete Dynamics in Nature and Society 3 (1999), no. 1, 57-63.

[2] S. S. Cheng and J.-Y. Lin, Stability criteria for a discrete reaction-diffusion equation, Far East J. Math. Sci. 6 (1998), no. 3, 425-435.

[3] S. S. Cheng and Y.-F. Lu, General solutions of a three-level partial difference equation, Comput. Math. Appl. 38 (1999), no. 7-8, 65-79.

[4] S.-N. Chow and J. Mallet-Paret, Pattern formation and spatial chaos in lattice dynamical systems. I, II, IEEE Trans. Circuits Systems I Fund. Theory Appl. 42 (1995), no. 10, 746-751, 752-756.

[5] A. Corduneanu, The asymptotic behaviour of the solution of some difference equations, Bul. Inst. Politeh. Iaşi. Secț. I. Mat. Mec. Teor. Fiz. 39(43) (1993), no. 1-4, 45-52.

[6] R. Medina and M. Pinto, Asymptotic behavior of solutions of second order nonlinear difference equations, Nonlinear Anal. 19 (1992), no. 2, 187-195.

[7] __ Nonlinear discrete inequalities and stability of difference equations, Inequalities and Applications, World Sci. Ser. Appl. Anal., vol. 3, World Sci. Publishing, New Jersey, 1994, pp. 467-482.

Rigoberto Medina: Departamento de Ciencias Exactas, Universidad de los Lagos, CASILla 933, OSORNO, CHILE

E-mail address: rmedina@ulagos.c1

Sui Sun Cheng: Department of Mathematics, Tsing Hua University, HSinchu, TAiWAN 30043, RePUBLIC OF CHINA 


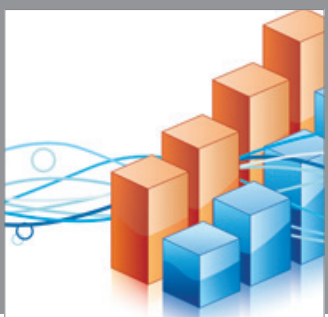

Advances in

Operations Research

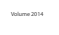

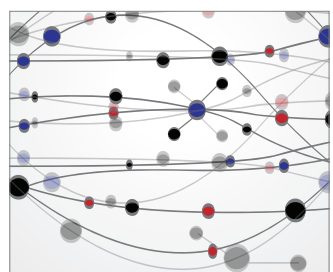

\section{The Scientific} World Journal
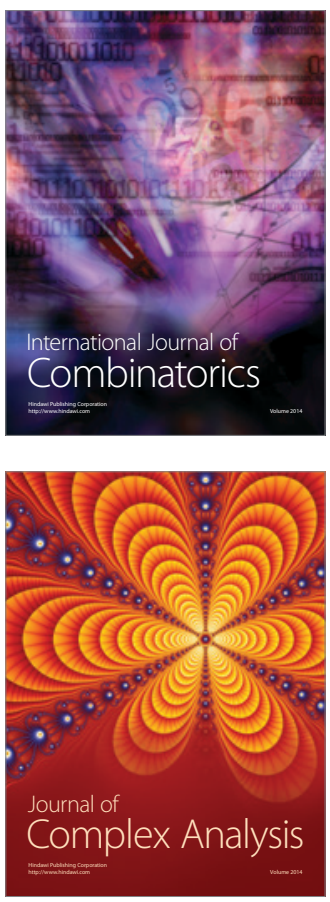

International Journal of

Mathematics and

Mathematical

Sciences
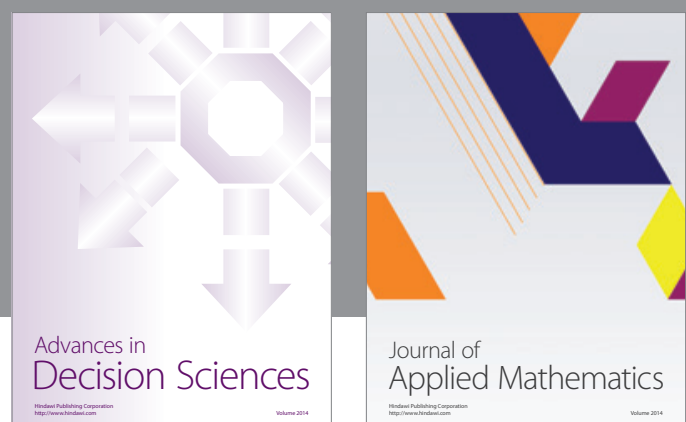

Journal of

Applied Mathematics
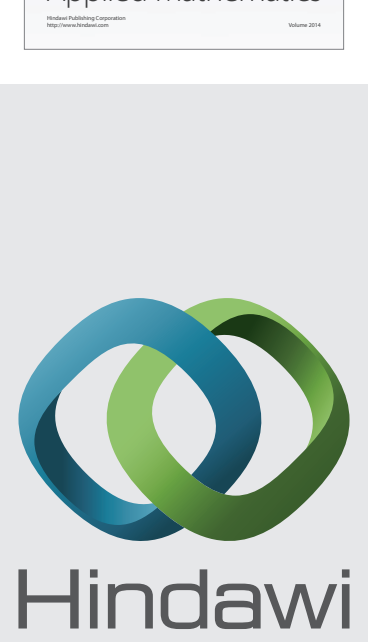

Submit your manuscripts at http://www.hindawi.com
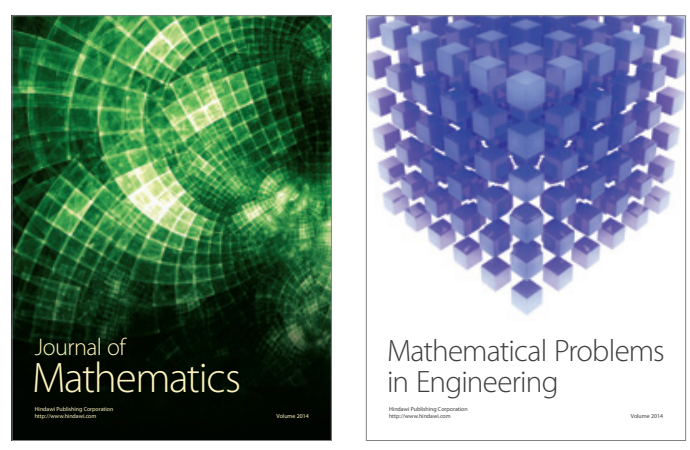

Mathematical Problems in Engineering
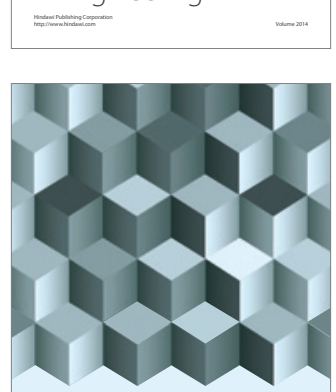

Journal of

Function Spaces
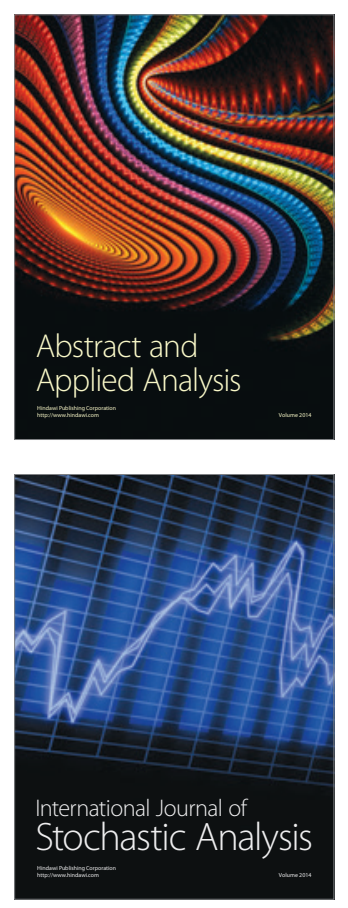

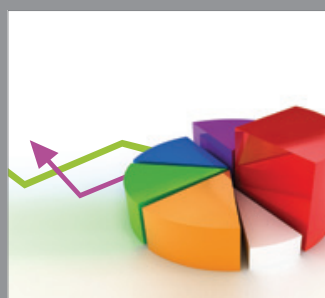

ournal of

Probability and Statistics

Promensencen
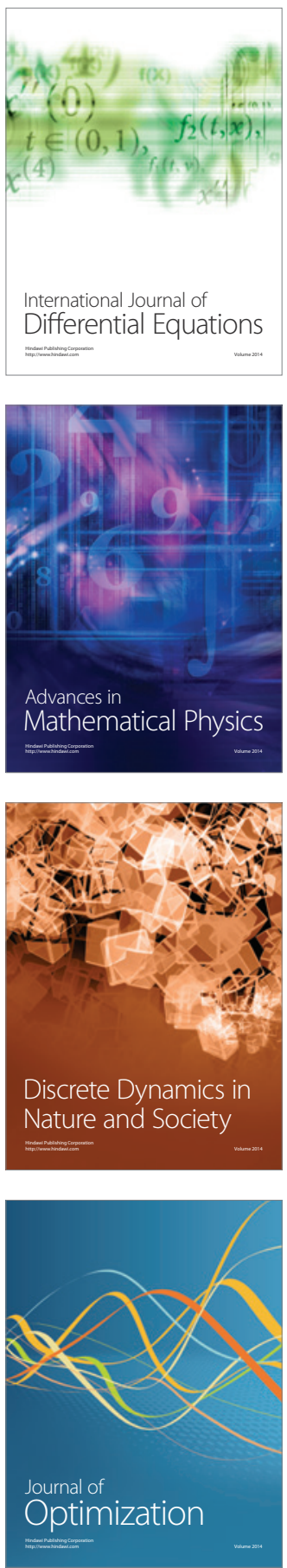